\title{
An efficient method for dichotic tape preparation
}

\author{
C. K. LEONG \\ Institute of Child Guidance and Development, University of Saskatchewan \\ Saskatoon, Saskatchewan, Canada
}

\begin{abstract}
The paper describes a simple but efficient method for dichotic tape preparation. The system of instrumentation together with checks for synchrony of stimuli and interstimulus intervals is explained. Methodological issues of onset synchrony and lag effect are discussed.
\end{abstract}

From the deceptively simple but ingenious procedures pioneered by Broadbent and Kimura in dichotic tape preparation have evolved more sophisticated techniques as described by Irvine (1972), Rubino (1972), Studdert-Kennedy and Shankweiler (1970), Yates, Smith, Burke, and Keane (1969), and Carr (Note 1). In essence, the procedure is as follows: (a) tryout recording of test materials onto tapes with due regard for intensity and duration of the stimuli; (b) splicing of "best" stimuli from tryouts onto a master tape loop; (c) rerecording of best stimuli from tryout tapes or from the master tape loops through some kind of time control device to achieve synchrony on the final tape. In actual practice, these steps are arduous and timeconsuming. For example, the check of duration through a spectrogram and, more conveniently, through an oscilloscope is often difficult. The excursions above noise level for different phonemes embedded in different acoustic environments vary and make for difficulties in determining onset synchrony. The splicing of tapes can be a frustrating experience for the novice. There is also a paucity of information on the attempt to achieve synchrony. Rubino's (1972) method, though fairly detailed. raises some doubts as to the claim of onset synchrony of less than $5 \mathrm{msec}$ in the final tape. The minor flaw is his use of a tape speed of 1-7/8 in. per second (ips), which reduces sound fidelity. The major flaw is the reliance on the "instant stop" buttons for recording and the monitoring by ear to decide on the precise moment for disengaging the buttons to begin transcription. Thus, his very refined 5 - msec onset difference between pairs of stimuli is more characteristic of computercontrolled instrurientation. Irvine's (1972) method seems to achieve reasonable results, but could do with more details explaining the precise procedure. In neither case is there any indication of the technical

The writer would like to thank Dr. Burchard M. Carr of Oklahoma State University and W. Diachuk of the University of Alberta for their suggestions. and Professor A. J. Yates of the University of Western Australia and the Editor of Behavior Research Methods \& Instrumentation, for their comments on the earlier draft of this paper quality of the final tape, save the claim that synchrony is achieved. On the satisfactory solution of technical problems such as onset synchrony and verification of interstimulus intervals depend the results of any dichotic study, as emphasized by Yates (1972). The purpose of the present paper is to describe a simple and yet efticient method for dichotic tape preparation together with a check for synchrony of stimuli.

\section{SYSTEM OF INSTRUMENTATION}

The system of instrumentation for the construction of dichotic tapes consists of: (a) two high-quality dual channel tape recorders (Sony 777-4J) with solenoid-operated run and record functions and with tape lifters that can be deactivated when the machine is in stop mode; (b) a specially designed and constructed cuing or control device for precise time alignment of stimulus materials; (c) a variable timer (a Heathkit sine-square audio generator) interfaced with the control device to achieve the desired stimulus interval. This is operationally defined as the duration between the onset of phonation of a stimulus (e.g. digit) and the onset of phonation of the next stimulus. As will be shown, the "beginning" of a stimulus is not easy to determine. A slight moditication is made to the first tape recorder by removing its Channel 2 input amplifier connection from the quarter-track head and connecting it to the Channel 1 half-track head. This enables the Channel 2 amplifier to be used as an amplifier for a voice-activated switch. The solenoid control provides the necessary remote control with the capability of being activated by voice impulses and deactivated after the desired interval from onset.

\section{BASIC IDEA}

The operational sequence in the tape preparation is as follows: (a) Preparation of tryout tapes by a phonetician with due regard to intensity and duration. An experienced speaker is trained to utter the stimulus signals, e.g., digits, at an even intensity as monitored on a VU meter and to release the final 


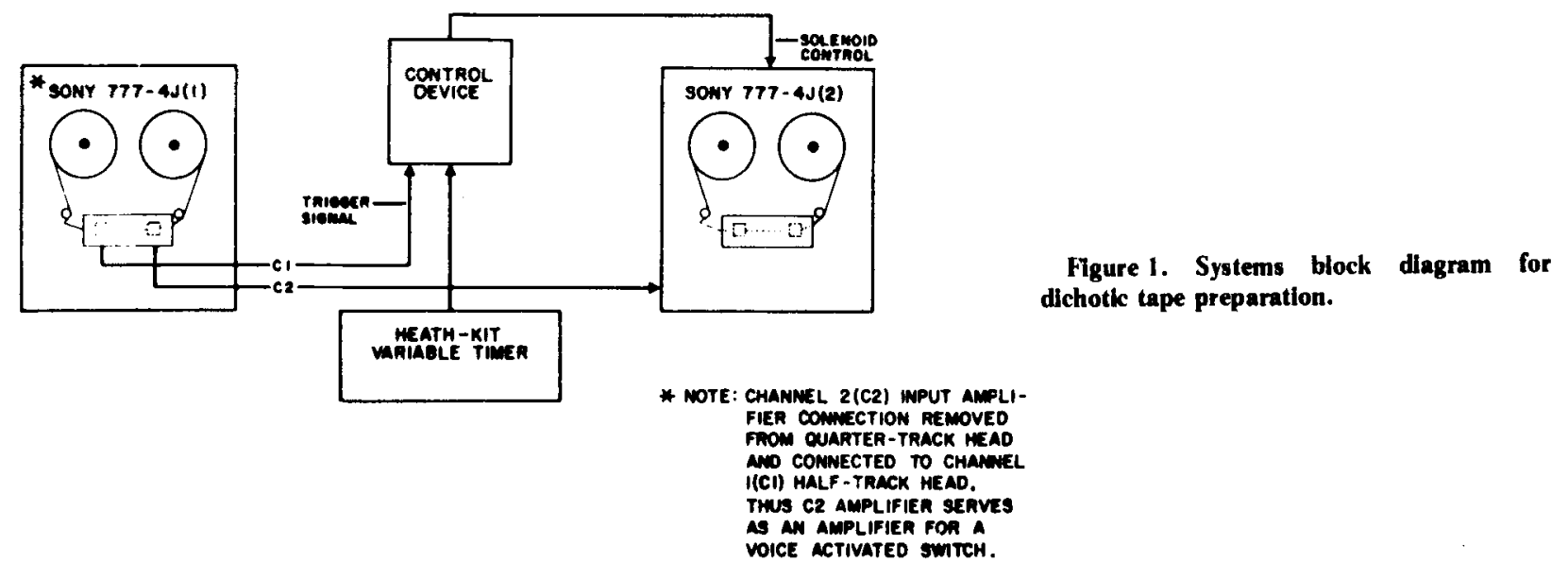

stops. Each digit is recorded a number of times, and the recordings are played through a Brüel and Kjaer sound-pressure-level recorder to obtain a record of the intensity of each digit. One example of each digit is chosen so that the final list contains only digits of about the same intensity. Imbalance in intensity is equalized to $75 \mathrm{~dB}$ SPL $\pm 1 / 2 \mathrm{~dB}$ as measured by the sound-pressure equipment for a $1.000-\mathrm{Hz}$ calibration tone equated to the intensity of the vocalic portion of the stimuli. (b) Recording of best samples from tryout tapes above onto one track of a second or master tape in such a way that the stimuli (digits, for example) to be used sequentially in the same series will follow one from the other at a speed slower than that required in the final tape. Take as an example the three-digit pair 5-9-8, in Track 1 , and 6-1-2, in Track 2. If the interstimulus interval is $1 \mathrm{sec}$, all that is required for the master tape is recording at a constant rate, separating the immediately adjoining digits in the sequence in the same track (5-9 or 9-8) greater than $1 \mathrm{sec}$. Other than this steady pace, no precise interval is needed at this stage. In practice, the 3- to 5-sec separation time is monitored on a Hunter decade interval timer. Also, the corresponding stimuli for Track $2(6-1-2)$ are recorded on the same track of the master tape. This is done by recording all the series for Track 1 first and then all the series for Track 2, or by alternating each corresponding series between Track 1 and Track 2 (such as 5-9-8 followed by 6-1-2) before going back to the next series in Track 1 , then that in Track 2. This latter method of alternating between tracks is more efficacious. (c) When the stimuli of matched intensity and duration within tolerance limits are properly recorded as in (b) onto a master tape, the latter is set in the first tape recorder in readiness for rerecording onto the two tracks of the second recorder to achieve onset synchrony. The interfacing of the two dual-channel recorders (the control device and the Heathkit timer) is shown in the systems diagram in Figure 1. Power supply and control logic diagrams are shown in Figures 2 and 3, respectively.

\section{RECORDING OF STIMULI AND CHECKING}

The basic principle is to use the voice impulses of the prerecorded stimuli on the master tape to activate, via the control device interfaced with the Heathkit timer, and thence the solenoid control, the second tape recorder which will thus start slightly ahead of the actual digits to be recorded. As the operation advances, with the desired interstimulus interval set on the timer linked to the control device, the latter "senses" the digits one at a time from the amplitication on Channel 1 . This sensing enables the second recorder to start, overcome inertia, pick up speed, and attain steady state by the time the actual information arrives from the modified playback head of the matched Sony $777.4 \mathrm{~J}$ and with a tape speed of 7.5 ips. This use of the voice activation and deactivation and the adjustable Heathkit timer for varying time intervals enables the digits to be recorded within the time duration specified. When recording of each series on Track 1 of the second tape recorder is completed as detailed, the second tape is wound back to specified marks on both the tape and recorder deck for approximate alignment in readiness for synchrony. The same process is repeated for the recording of the corresponding series on Track 2 in synchrony with those digits already recorded on Track 1. When this is accomplished for one series-pair $\begin{gathered}5-9-8 \\ 6-1-2\end{gathered}$, the same procedure is repeated with the next series on Track 1 , which is paired with its corresponding series on Track 2 . Preceding the test items, sufficient tape length is allowed for the insertion of instructions binaurally while adequate response time is given between each series. This response time can be augmented by stopping the tape recorder manually when it is found that the subject needs more time to make a verbal report.

Following completion of the final tape, synchrony can be tested on a polygraph (Hewlett-Packard 760 preamplifier with two-channel printout at varying rates from 0.25 to $100 \mathrm{~mm} / \mathrm{sec}$ ). The use of the apparatus is practical and provides an easy-to-read 


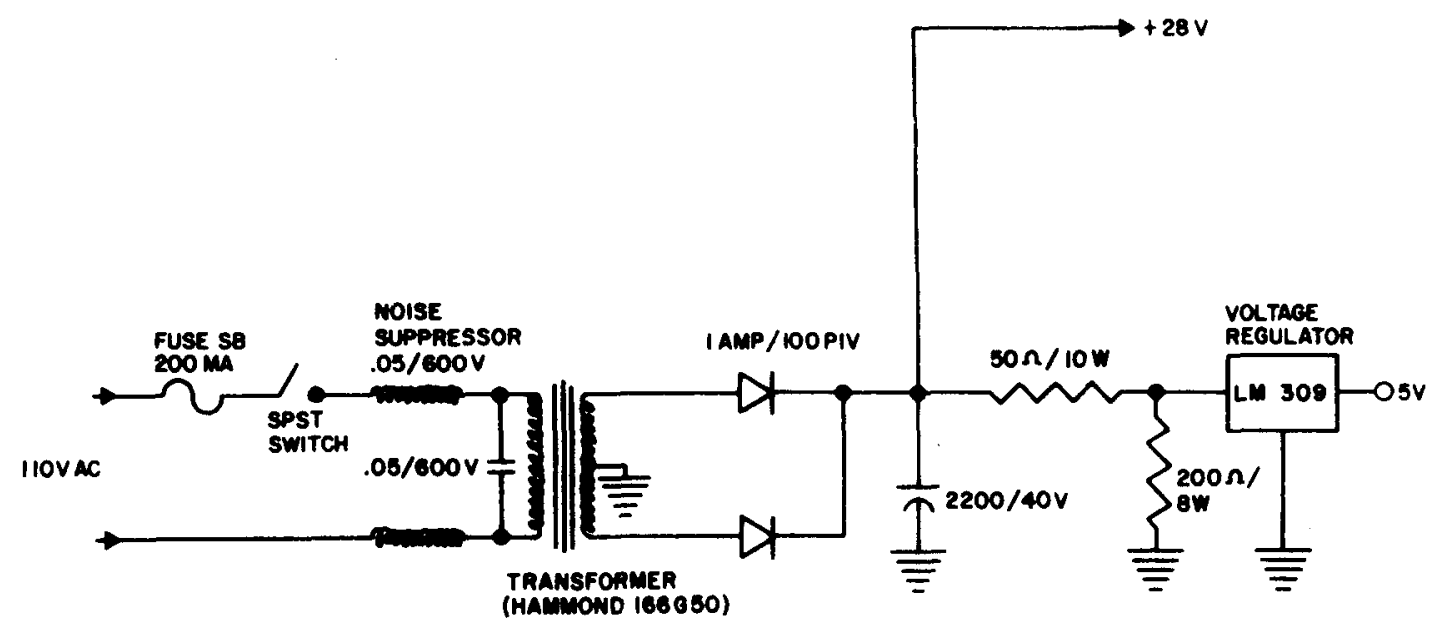

\section{POWER SUPPLY DIAGRAM}

Figure 2. Power supply diagram for system of instrumentation.

printout for comparisons of onset, offset, and interstimulus intervals of stimuli. From the tapes, synchrony falls within $5 \mathrm{msec}$ and interstimulus intervals are held constant within the same tolerance limit. An example of the verification is shown in Figure 4 .
With equalizing of intensity of stimuli on the master tape, another attempt should be made to further ensure equal intensity between the channels on the final tape. One way to achieve this is to direct the stimulus materials to a compressor amplifier or to play the final tape through a Shure audio-mixer. The

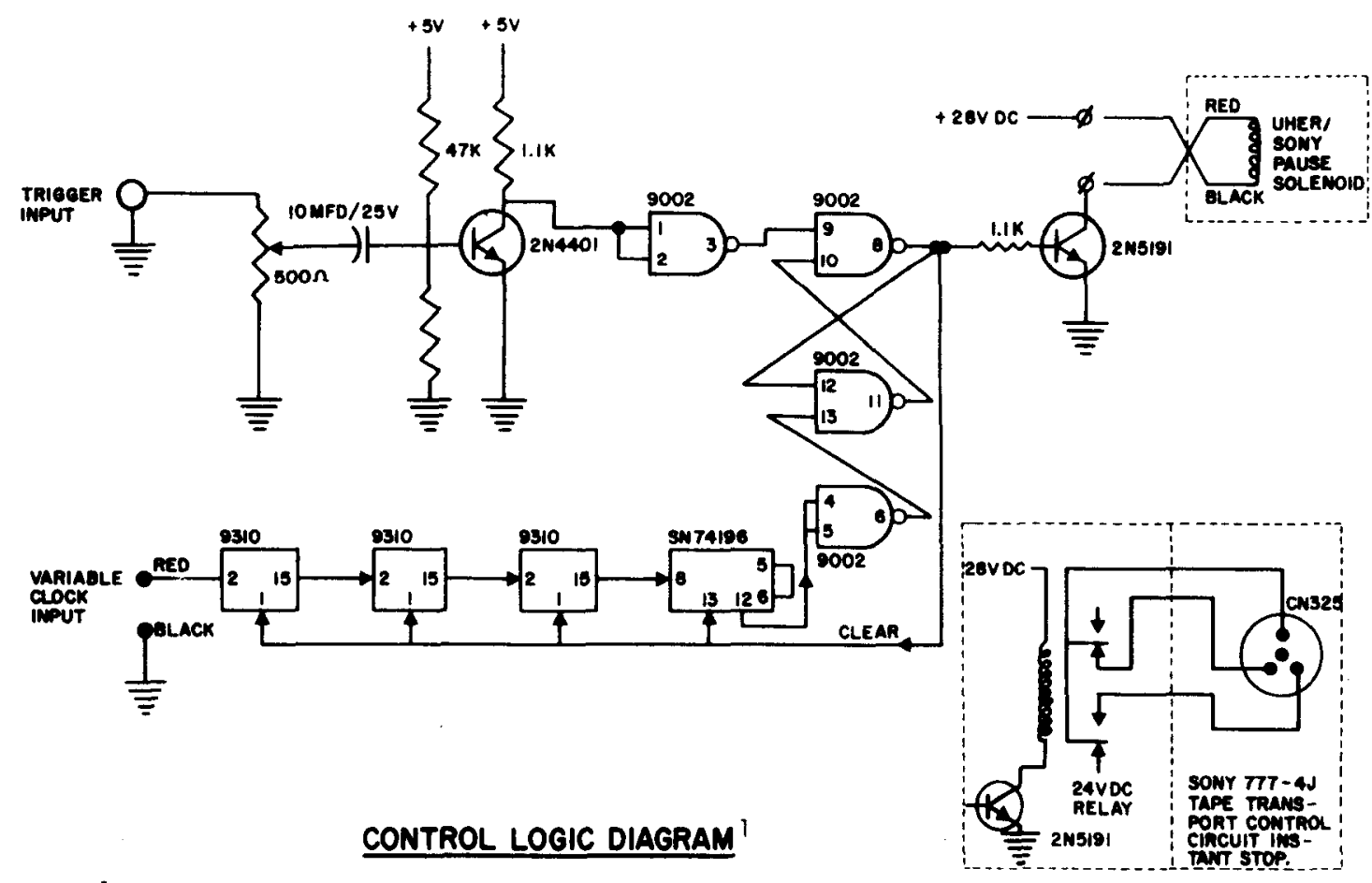

Inset shows modification from Uher 8000 to Sony 777-4J.

Figure 3. Control logic diagram for system of instrumentation. 

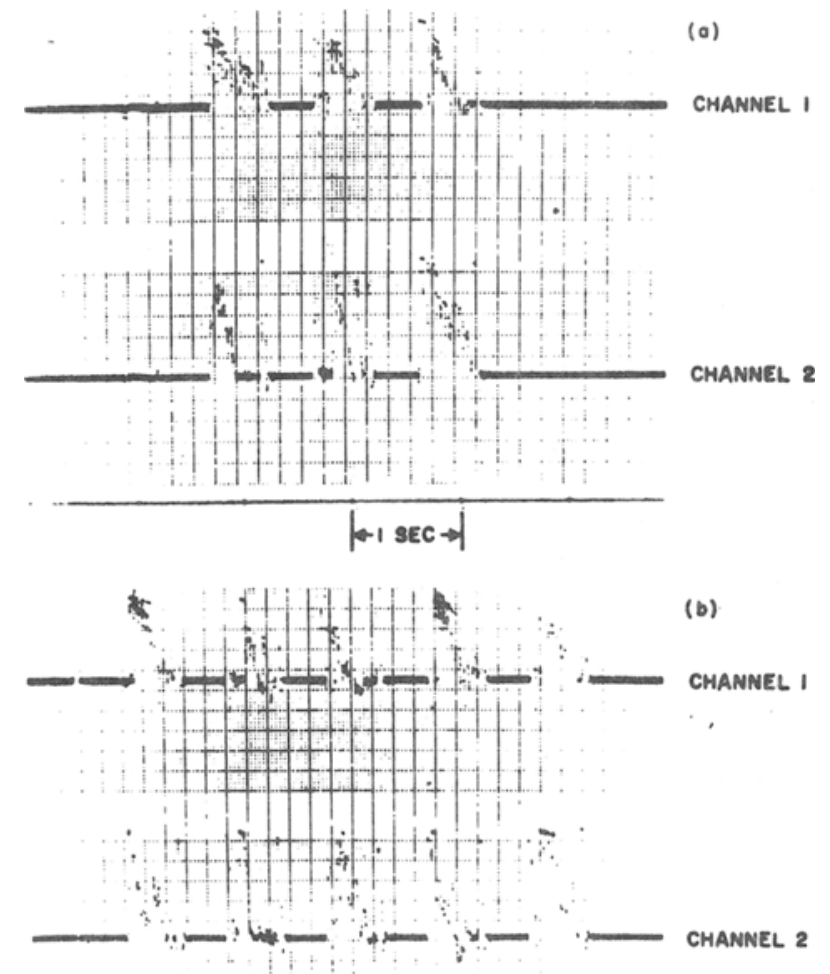

k-1 secold

Figure 4. Sample polygraph tracings on a reduced scale (ortginal printout on $25 \mathrm{~mm} / \mathrm{sec}$ ) of dichotic digits to show synchrony, interstimulus interval, and duration of digit sequences. Tracings in top record show 9.3.1 in Channel 1 and 8.7.9 in Channel 2 (from top down). Tracings in bottom record show 2-7-4-2-3 and 5-6-1-4-2 in Channel 1 and Channel 2, respectively.

question of intelligibility of dichotic stimuli is a vexed one. An attempt is made to ensure, on the part of the subjects, perfect intelligibility at $75 \mathrm{~dB}$ SPL of all monaural individual digits and of samples of dichotic pairs. The fusion effect demonstrated by Day (Note 2), such that dichotically presented nonsense words "banket," given to one ear, and "lanket," to the other ear, are fused to produce "blanket," provides some insight into the combination of speech information centrally. As far as could be ascertained, the fusion effect of the dichotic pairs studied is negligible.

\section{SUMMARY AND DISCUSSION}

To summarize the methodological aspects of dichotic tape preparation, it is pertinent to reflect on some difficulties encountered. Considerable operational skills are required in making dichotic tapes, given the equipments described. Rerecording is often required to ensure that the stimuli fall within reasonable time limits (e.g., less than $2.5 \mathrm{sec}$ for a three-digit series). However, once well versed with the system, the actual preparation of a 15-itemed tape with three-digit pairs on the average should take about 6 to $8 \mathrm{~h}$. One difficulty relates to the limitations imposed by the mechanical aspect of even high-quality tape recorders, which in turn affect rates of presentation. It takes some time for a tape recorder to get started, pick up speed, attain the steady state required to make a recording without disturbing the acoustic patterns. The time required is estimated at around $180 / 200 \mathrm{msec}$ with the matched Sony $777-4 \mathrm{~J}$. Added to this is the duration needed to utter a syllable, which clusters around $350 / 400$ msec within a range of 350 to $500 \mathrm{msec}$, as found by the writer. This compares with the finding of Yates et al. (1969) of variations between 407 and $553 \mathrm{msec}$ and with that of Studdert-Kennedy and Shankweiler (1970) of a range of 300 to $500 \mathrm{msec}$ for natural CVC syllables. Some variation is needed for maximum intelligibility as syllables have natural acoustic durations. Thus, for a spoken syllable or a digit to be recorded with the present system, or for that matter the other systems outlined, the operation takes about $550 / 600$ msec. This raises doubts as to the claim or the precise meaning of the often-mentioned "two pairs per second" in the literature. From empirical findings, the only acceptable explanation of the assertion is that of "the interval between the beginning of one digit and that of the next was 1 sec" (Broadbent \& Gregory, 1961, p. 106).

The "beginning of one digit" is explained by Studdert-Kennedy and Shankweiler (1970) as the first excursions above noise level in an oscillographic record, that is sustained and followed by clear periodicity. In actual practice, the instant of onset for a monosyllable is generally difficult to determine since the beginning of phonation is not marked by a single event having a conspicuous acoustic correlate which is easily displayed on a cathode ray tube of a two-channel storage oscilloscope. This difficulty is minimized by the use of test materials which begin with plosive consonants. By so doing, the point where the speech signal departs from ambient background noise to a fast rising aperiodic excitation of voicing can be agreed upon consistently. The departure from ambient back ground noise serves as the operationally defined point of "onset." The finding by Berlin, Lowe-Bell, Cullen, Thompson, and Loovis (1973) and Lowe, Cullen, Berlin, Thompson, and Willett (1970) of differential perception affected by $30-$ to $60-\mathrm{msec}$ lag effect further highlights the problems related to onset synchrony.

The present system of instrumentation also shows that the acoustic qualities of phonemes are important. During the preparation of tapes, it was found that the control device worked well with pure tones which would activate and deactivate the second tape recorder precisely within the desired time interval. 
With speech sounds, sound streams in different phonemic environments and with different signal-tonoise ratio might trigger off the second recorder ahead of the stipulated time, thus throwing synchrony into slight disarray. This can happen when the intensity is too high, such as with the immediate onset of voicing in "one" as compared with "five." It can happen also with decreased signal-to-noise ratio, as with a compressed tape compressed on a BA-43/45 AGC Program Amplifier Unit 11455. This unexpected "filtering" is double-edged. On the one hand, it alerts the researcher to the need to carefully control intensity and other acoustic qualities prior to attempting synchrony. It also points to the vulnerability of an electronic/mechanical system short of computer control in response to complex speech codes. As demonstrated with discrete pure tones, were the mostly monosyllabic stimuli (speech segments) strictly linear, the instrumentation would work perfectly to specification. As it is, speech is also multidimensional and the experimentation with the dichotic system provides some indirect evidence for this. Up till now, there has been considerable effort in improving the technical quality of dichotic tapes. Perhaps the role of the ipsilateral pathways and the "psychological moment" of the lag effect in the context of speech lateralization (see Belin et al., 1973) should deserve greater attention. From the evidence that dichotic listening involves an additive central process which combines speech information from both ears (Cullen. Thompson, Hughes, Berlin, \& Samson, 1974), the differential roles of acoustic, phonetic, and linguistic parameters should also be further investigated.

\section{REFERENCE NOTES}

1. Carr. B. M. A new system for preparing dichotic listening tapes. Paper presented at the annual convention of the American Speech and Hearing Association. Chicago, November 1969.
2. Day, R. Digit-span memory in language-bound and stimulus-bound subjects. Haskins Laboratories: Status Report on Speech Research. SR-34. 1973, 127-139.

\section{REFERENCES}

Berlin. C. I., lowe-Bell, S. S., Cullen, J. K., JR., Thompson, C. L., \& Loovis, C. F. Dichotic speech perception: An interpretation of right-ear advantage and temporal offset effects. Journal of the Acoustical Society of America, 1973. 53. 699.709.

Broadbent. D. E., \& Gregory. M. On the recall of stimuli presented alternately to two sense organs. Quarterly Journal of Experimental Psychology, 1961, 13, 103-109.

Cullen, J. K.. Jr., Thompson, C. L., Hughes, L. F., Berlin, C. I..\& SAmson, D. S. The effects of varied acoustic parameters of performance in dichotic speech perception tasks. Brain and Language. 1974, 1, 307-322.

IRVINE. J. W.Handedness preference, manual dexterity, ear asymmetry in dichotic listening and grade 2 reading proficiency. Unpublished doctoral dissertation, University of Alberta, 1972.

Lowe, S. S., Cullen, J. K., Jr., Berlin, C. I., Thompson, C. L., \& WilletT, M. E. Perception of simultaneous dichotic and monotic monosyllables. Journal of Speech and Hearing Research, 1970, 13, 812-822.

Rubino. C. A. A simple procedure for constructing dichotic listening tapes. Cortex, 1972, 8, 335-338.

Studdert-Kennedy, M., \& Shankweiler, D. Hemispheric specialization for speech perception. Journal of the Acoustical Society of America, 1970, 48, 2, Pt. 2, 579-594.

YATES, A. J. Technical, methodological and theoretical problems in dichotic stimulation research. Australian Psychologist, 1972, 7, 2-19.

Yates, A. J., Smith, P. J., Burke, B. D., \& Keane, M. A. A technique for the construction of discrete dichotic stimulation material. Behavior Research Methods \& Instrumentation, $1969,1,257-258$.

(Received for publication February 12, 1975; revision received April 1, 1975.) 